\title{
Los estilos de liderazgo y su efecto en la satisfacción laboral
}

\section{Leadership styles and their effect on job satisfaction}

Kiana Annabel Añazco Camacho

Escuela Superior Politécnica del Litoral, Ecuador

Rosalva Paulina Valdivieso Salas

Escuela Superior Politécnica del Litoral, Ecuador

Óscar William Sánchez Córdova

Escuela Superior Politécnica del Litoral, Ecuador

María Auxiliadora Guerrero Bejarano, MBA

Universidad Internacional del Ecuador, Ecuador

Autor para correspondencia: kanazco21@gmail.com,pauvy06@hotmail.com, o_sanchezcordova@hotmail.com,_maguerrerobe@uide.edu.ec

Fecha de recepción: 23 de abril de 2018 - Fecha de aceptación: 30 septiembre de 2018

Resumen: En el presente trabajo se hace una recopilación de las lecturas relacionadas al liderazgo y la satisfacción laboral. Se han revisado distintos estudios efectuados sobre estos temas, en donde esta relación es motivo de una satisfacción elevada en los empleados, sin considerar la empresa u organización en que se labore, sea esta: estatal o privada, pequeñas, medianas o grandes industrias. También se probó el papel de la satisfacción y la relación con los diferentes estilos de liderazgos como el transformacional y la organización. La intención de este ensayo es presentar como el liderazgo influye de forma positiva en la satisfacción laboral, lo cual es una realidad que se ha visto reflejada en distintas partes del mundo, por lo tanto, se desea difundir estos resultados en nuestro medio para que se tomen con mayor atención en nuestras organizaciones.

Palabras Claves: liderazgo; transformacional; satisfacción laboral; organizaciones

\begin{abstract}
In this work a compilation of the readings related to leadership and job satisfaction is made. Various studies have been reviewed on these issues, where this relationship is a reason for high employee satisfaction, regardless of the company or organization in which it is worked, be it state or private, small, medium or large industries. The role of satisfaction and the relationship with the different leadership styles such as the transformational and the organization were also tested. The intention of this essay is to present how leadership positively influences job satisfaction, which is a reality that has been reflected in different parts of the world, therefore, we want to disseminate these results in our environment so that take more attention in our organizations.
\end{abstract}

Key Words: leadership; transformational; job satisfaction; organizations 


\section{Introducción}

Acontecimientos que han marcado la historia de la humanidad han sido influenciados por personajes que pasaron a la eternidad por su capacidad de movilizar a mareas humanas hacia el cumplimiento de un objetivo común. Desde tiempos inmemoriales se han levantado figuras que por su casta de líderes llevaron a los suyos a lo más alto de su área de desempeño. En la ciencia, arte, milicia, religión, negocios y en muchos otros campos han surgido estos caudillos, cada uno con un estilo que lo diferencia de los demás. Bonaparte que por su férreo carácter pudo salir victorioso de varias contiendas bélicas, el pastor Martin Luther King un apasionado por la lucha en contra de la segregación y discriminación racial (Gomis, 2013), Ray Kroc cuya idea de transformar a McDonald's en una entidad mundial mostró su estirpe de un visionario de los negocios (Maxwell, 2007). El éxito de estas personas no solo se debe a las habilidades que poseían sino también al esfuerzo, entrega y compromiso que sus respectivos equipos de trabajo aportaban.

Para poder lograr que los miembros de una organización tengan esas características, ellos deben desenvolverse en un entorno que estimule el bienestar emocional y psicológico lo cual desencadena un alto nivel de satisfacción laboral que se traduce en rendimiento general para la organización. El presente artículo está orientado a la investigación de la relación que pudiere existir entre los distintos estilos de liderazgo y la satisfacción laboral indiferente de la industria o región geográfica. (Ramandeep, Vaishalli, \& Andotra, 2016)

\section{Estilos de Liderazgo}

El liderazgo es una habilidad que tiene una persona para dirigir, pensar, delegar e incentivar a las personas que están bajo su responsabilidad para realizar su trabajo de una manera adecuada, correcta y con responsabilidad, se puede decir que es la influencia que se ejerce sobre otra persona (Maxwell, 2007). Es necesario saber que existen diferentes tipos de líderes, ya que no todos actúan de la misma manera, identificar a cada uno de ellos ayudará a poder reconocer si es un líder apto para poder cumplir con los requerimientos de la organización o encasillarlo en otro tipo (Olcer, 2015).

El líder desempeña un papel clave en la satisfacción laboral, destacando varios estilos de liderazgos que se han descrito desde el marco de las empresas y organizaciones contemporáneas. Los estilos de liderazgo se han clasificado en varios tipos de los cuales los más conocidos o estudiados son los autocráticos y democráticos (Lewin, Lippit, \& White, 1939) y transformacional y transaccional (Bass, 1985). Se debe tener en cuenta que los estilos de liderazgo existen interrelaciones entre los líderes y sus seguidores, siendo esto lo más importante para mejorar la eficiencia y la satisfacción de los empleados. (Ramandeep, Vaishalli, \& Andotra, 2016). Estos estilos de liderazgo son considerados como factores importantes que influyen en el desempeño de la organización, sea con gerentes o empleados (Hunjra, Chani, Aslam, Azam, \& Rehman, 2010).

\section{El liderazgo transaccional}

Este líder busca el beneficio para los empleados y para la empresa. En este tipo de liderazgo suele dar bonificaciones o incentivos a los empleados por labores realizadas motivándolos a realizar mejor su trabajo. Burns, quién desarrolló la teoría que incluye este estilo de liderazgo afirma que "El liderazgo transaccional ocurre cuando una persona se conecta con otras con la 
intención de intercambiar valor. Cosas que podrían ser de naturaleza económica, política o psicológica." (Burns, 1978).

\section{Liderazgo Transformacional}

Se centra como líder en la satisfacción de sus subordinados, aumentando la productividad y eficacia. Estos líderes inspiran a su equipo a ser más productivos y exitosos, llevándolos a trascender su propio interés por un nivel superior. Según Burns el estilo de liderazgo transformador "ocurre cuando una o más personas se involucran con otros en tales una forma en que los líderes y seguidores se elevan entre sí a un mayor nivel de motivación y moralidad" (Burns, 1978).

\section{Liderazgo burocrático.}

Se encuentra en ámbitos empresariales o políticos, más que ser un líder es un jefe, muchas organizaciones tienen este tipo de líderes que son ubicados en ese sitio por sus estudios o experiencia en el área de trabajo mas no por sus habilidades de liderar o manejar empleados, por lo que se puede confundir y no ser considerado como parte del equipo de trabajo; aunque la mayoría de organizaciones prefieren tener jefes y no líderes, debido a la necesidad que los empleados cumplan órdenes y no necesariamente aporten con ideas (Gómez Ortiz, 2008).

\section{Liderazgo Carismático}

Esta persona en el ámbito empresarial podría no ser muy atractiva, ya que los empleados pueden confundir la amabilidad con amistad, corriendo el riesgo que no tomen en serio las órdenes y no realicen su trabajo con responsabilidad, este tipo de líder busca la satisfacción de su equipo de trabajo, reduciendo los problemas entre líder y su equipo de trabajo debido a que se sienten escuchados al ser tratados de una forma amable creando así un ambiente amigable (Weber, 1946). Un buen líder debe estar comprometido con su equipo de trabajo con su bienestar, felicidad y motivación (Olcer, 2015), que motive a su equipo a mejorar y tener sus objetivos claros. Además debe de tener credibilidad, saber comunicarse con los demás, tener seguridad en la manera de actuar, evitar dañar la autoestima de su equipo, motivándolos, y demostrando la habilidad de delegar con responsabilidad y compromiso.

La relación entre algún estilo de liderazgo y la satisfacción laboral ha sido investigada en distintas industrias y diferentes países de varios continentes. En las siguientes líneas se detallará algunos de los estudios que se han realizado. En el sector educativo de Pakistán se llevó a cabo un estudio donde se confirma que el liderazgo transformacional tiene una asociación positiva con la satisfacción laboral, esto quiere decir que el líder a través de su comportamiento inspirador y motivador puede inducir cambios psicológicos en los miembros de la organización. Por su parte el liderazgo transaccional guarda una relación negativa con la satisfacción laboral, lo que significa que estos tipos de líderes están más preocupados por lograr los objetivos a través de recompensas o castigos y tienen menos énfasis en la motivación de los miembros de la organización (Saleem, 2015). 
Uno de los mitos acerca del liderazgo es que ésta habilidad es exclusiva para los empresarios de una organización (Maxwell, 2007). En la atmosfera de las personas que ejercen una función de mando medio (supervisores, analistas,inspectores,etc.), uno de sus aspectos mas importantes es la gestión de recursos y el que acapara la mayor importacia es el del personal (Thomas, 2012). Se han realizado investigaciones del liderazgo en mandos medios, como el llevado acabo en la Argentina (Omar, 2011), quien analizó las asociaciones de las diferentes matices del liderazgo transformador (consideración, motivación, influencia y estimulación intelectual), la confianza del supervisor, la satisfacción laboral y de la vida en general.Omar identifico que los cuatros componestes del liderazgo transformador se vincula moderadamente con la satisfacción laboral, pero la mayor correlación se haya en la estimulación intelectual $(\mathrm{r}=0.52$; $\mathrm{p}<0.01)$ e influencia idealizada $(\mathrm{r}=0.46 ; \mathrm{p}<0.01)$. Añade tambien que la confianza en el supervisor se correlaciona tanto con la satisfacción laboral $(r=0.48 ; p<0.01)$ como la vida general $(\mathrm{r}=0.39 ; \mathrm{p}<0.01)$. Como hemos podido notar el estilo de liderazgo tranformacional ha sido aplicado en dististos paises (Argentina y Pakistan ) con diferencias culturales, sociales, desarrollo económico y tecnológico mas sin embargo se ha llegado a la misma conclusión de su impacto positivo en la satisfacción laboral.

En otro estudio en el servicio de la educación se realizó en el Ecuador en la Escuela Superior Politécnica Agropecuaria de Manabí (ESPAM). En la investigación realizada se determinó que en este centro estudiantil se aplica el liderazgo de Dirección en equipo, la cual promueve equilibrio entre la orientación a las personas y a los resultados, adaptándose a diferentes situaciones e individuos al igual que el liderazgo situacional que desencadena un buen ambiente de trabajo (Alava Rade \& Vega Chica, 2017). En los servicios de la salud se ha estudiado tambien el efecto del liderazgo en la satisfacción laboral. (Boamah, Spence Laschinger, Wong, \& Clarke, 2017), investigan los efectos del liderazgo transformacional en la satisfacción en el trabajo de enfermeras y la seguridad de los pacientes en Ontario. Los hallazgos de este estudio muestran el importante papel que juegan los líderes transformadores en la mejora de la calidad del ambiente de trabajo para las enfermeras y mejoran la seguridad del paciente aumentando la satisfacción de las enfermeras en el trabajo. Resultados similares fueron encontrados en una casa de salud en Colombia (Contreras Torres, Espinal G., Pachón E., \& González R. , 2013), el liderazgo y la satisfacción laboral muy relacionados por la motivación intrínseca.

El liderazgo auténtico ha sido también sometido a estudio, Olaniyan \& Hystad (2016), investigaron los efectos directos e indirectos de este tipo de liderazgo. Un líder auténtico muestra la confianza, la esperanza, el optimismo, las emociones positivas, la transparencia relacional y una orientación moral y ética hacia el futuro. (Avolio, Gardner, Walumbwa, Luthans, \& May, 2004). El estudio de Olaniyan \& Hystad (2016), fue realizado a trabajadores de la industria de reabastecimiento de envío de petróleo y gas oriundos de Noruega, Filipinas y entre otras nacionalidades (europeas, asiáticas /Australia). Los resultados arrojados fueron que los trabajadores que percibían a sus jefes como auténticos estaban más satisfechos y con menor inseguridad laboral e intención de abandonar la empresa. Olaniyan \& Hystad concluyen tambien que el liderazgo auntentico ejerce tambien influencia indirecta a un conjunto de variables psicológicas por ejemplo la autosuficiencia,optimismo,esperanza y resilencia.

Las entidades públicas han sido objeto de estudio acerca del impacto que ocasiona el liderazgo en la satisfacción laboral de los servidores públicos. Así tenemos el trabajo llevado a 
cabo por (Peñarreta Cuenca, 2014) en el Gobierno Autónomo descentralizado (GAD) del Municipio de Loja. En esta institución, la autora advierte que prevalecen los estilos equilibrado, empobrecido y club social en esta entidad. Además, encuentra disensión por parte de los empleados con respecto a los modelos de liderazgo que en la organización se implementan. Peñarreta observa que en esta institución los estilos de liderazgo son valorados de distinta manera entre los empleados, lo cual complica el logro de los objetivos de la organización.

Que relación puede existir entre la percepción como lideres y como realmente nos evaluan los subordinados, fue la interrogante que planteron (Amundsen \& Martinsen, 2014). Estos investigadores estudiaron el efecto del acuerdo entre el yo y el otro, en el empoderamiento del liderazgo sobre la efectividad del lider, la satisfaccón del trabajo y la inteción de rotación con una muestra de 50 lideres municapales de noruega. Los resultados obtenidos demuestran que los lideres que sobrestimaron su empoderamiento en liderazgo tienen subordinados con una menor satisfacción en el trabajo y una alta intención de rotación del trabajo. Por otro lado los líderes que subestimaron su liderazgo fueron mas definidos como efectivos por sus superiores. Estos resultados nos hace pensar de la importancia del feedback que debe existir entre el lider y sus seguidores para que se pueda nutrir de los comentarios de ellos y evitar sufrir de la denominada ceguera del liderazgo y mas bien sirva de aporte a la toma de deciones sabias para la solución de problemas (Panasiuk, 2015).

\section{Conclusiones y Recomendaciones}

En cada una de las investigaciones a las que hemos consultado, se ha determinado que el liderazgo es una fuerza que impulsa la satisfacción laboral. En dististos sectores (educación, salud, hidrocarguro y entidades gubernamentales) en paises de America ,Europa y Oceania se ha demostrado esta fuerta relación entre ambas variables. Es de suma importancia excelentes niveles de satisfacción laboral en las organizaciones ya que estimula el compromiso de los empleados (Ahmad \& Ibrahim, 2015). Teniendo ya el conocimiento de los efectos del liderazgo es indispensable el desarrollo de líderes en las organizaciones (con y sin fines de lucro) en países como el nuestro que están en plena carrera hacia el progreso. En este proceso de desarrollo del liderazgo en nuestra Latinoamérica se debería asegurar en primera instancia la concepción correcta de su significado y poner fin al síndrome del mesías que por mucho tiempo ha estado instaurado en nuestros pueblos. Como recomendaciones que surgen de este ensayo planteamos:

Emular el trabajo de Amundsen \& Martinsen apliacando el acuerdo entre el yo y el otro, en nuestro pais en distintas industrias y estudiar las percepciones de nuestros lideres con repecto asi mismo y las que tienen sus subordinados en referencia a su desempeño en liderazgo. El liderazgo y la satisfacción laboral según la cohorte generacional en Ecuador, surgue como tema interesante de investigación, para indagar si en nuestro medio existe diferencias en el modo de como responden los individuos de distintas épocas a los diferentes estilos de liderazgo. Determinar la brecha entre el Ecuador y paises de la region en lo que se refiere al liderazgo y su impacto en la satisfacción laboral. Es una investigación que podria aportar mucho al fortaleciomiento de esta habilidad a personal de mandos medios, jefes y Gerentes del pais. 


\section{Bibliografía}

Ahmad, H., \& Ibrahim, B. (2015). Leadership and the Characteristic of Different generational Cohort towards Job Satisfaction. Procedia - Social and Behavioral Sciences, 14-18.

Alava Rade , F. E., \& Vega Chica, M. L. (2017). Incidencia del Liderazgo Situacional en el Índice de Satisfacción Laboral de los Empleados de la ESPAM. Manabi: INNOVA.

Amundsen, S., \& Martinsen, Ø. L. (2014). Self-other agreement in empowering leadership: Noruega: Elsevier.

Avolio, B., Gardner, W., Walumbwa, F., Luthans, F., \& May, D. (2004). Unlocking the mask: A look at the process by which authentic leaders impact follower attitudes and behaviors. The Leadership Quarterly, 801-823.

Bass, B. (1985). Leadership and Performance. N. Y.: Free Press,.

Boamah, S., Spence Laschinger, H., Wong, C., \& Clarke, S. (2017). Effect of transformational leadership on job satisfaction and patient safety outcomes. Nursing Outlook, 1-10.

Burns, J. M. (1978). Leadership. New York, NY.

Contreras Torres, F., Espinal G., L., Pachón E., A. M., \& González R. , J. (2013). Burnout, liderazgo y satisfacción laboral en el personal asistencial de un hospital de tercer nivel en Bogotá. Diversitas- Perspectivas en Psicología , 80.

Gómez Ortiz, R. A. (junio de 2008). El liderazgo empresarial para la innovación tecnológica en las micro, pequeñas y medianas empresas. Barranquilla, Colombia.

Gomis, J. (2013). Antologia Martin Luther King Un sueño de igualdad. Madrid: Catarata.

Hunjra, A. I., Chani, M. I., Aslam, S., Azam, M., \& Rehman, K. U. (2010). Factors effecting job satisfaction of employees in Pakistani banking sector. African Journal of Business Management, , 2157-2163.

Lewin, K., Lippit, R., \& White, R. K. (1939). Patterns of Aggressive Behavior in Experimentally Created Social Climates.

Maxwell, J. c. (2007). Desarrolle el Lider que está en usted. Nahville, Tennessee: Caribe.

Olaniyan, O. S., \& Hystad, S. W. (2016). Employees' psychological capital, job satisfaction, insecurity, andintentions to quit: The direct and indirect effects of authenticleadership. Madrid: Elsiever.

Olcer, F. (23 de Febrero de 2015). An Empirical Study on the relationships among tranformational leadership dimensions, Job atisfaction an organizational. Obtenido de https://www.liderazgo.co/contacto/ 
Omar, A. (2011). Liderazgo Transformador y satisfacción laboral: El rol de la confianza en el supervisor. Scielo, 129-137.

Panasiuk, A. (2015). Deciciones que cuentan Principios para tomar deciciones económicas que te cambiarán la vida. Nashville: Grupo Nelson.

Peñarreta Cuenca, D. S. (6 de Marzo de 2014). La influencia de los estilos de liderazgo en los niveles de satisfacción laboral de los empleados del GAD Municipal de Loja. Loja, Loja, Ecuador.

Ramandeep, K., Vaishalli, \& Andotra, N. (2016). Leadership Styles and Job Satisfaction among Employees. International Journal on Leadership, 34-35.

Saleem, H. (2015). The impact of leadership styles on job satisfaction and mediating. Pakistan: Elsevier .

Thomas, C. J. (2012). Guia Básica para Mandos Intermedios y Jefes de equipo . Madrid: Fc Editorial .

Weber, M. (1946). The sociology of charismatic authority. Essay in Sociology. New York. 